\title{
Benign Ovarian Sex Cord-Stromal Tumor
}

National Cancer Institute

\section{Source}

National Cancer Institute. Benign Ovarian Sex Cord-Stromal Tumor. NCI Thesaurus.

Code C6803.

A sex cord-stromal tumor arising from the ovary, without metastatic potential. 